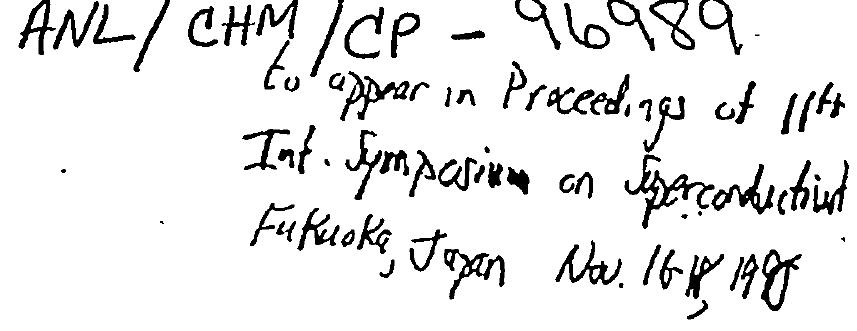

\title{
Magnetic imaging of interlayer Josephson vortices
}

\author{
J.R. Kirtley ${ }^{1}$, K.A. Moler ${ }^{2}$, J.A. Schlueter, J.M. Williams ${ }^{3}$, D.G. Hinks ${ }^{3}$, T.W. Li ${ }^{3}$, G. Villard ${ }^{4}$, \\ and A. Maignan ${ }^{4}$ \\ ${ }^{1}$ IBM Research Division, Yorktown Heights, NY 10598, USA \\ ${ }^{2}$ Dept. of Applied Physics, Stanford University, Stanford, CA 94305, USA \\ ${ }^{3}$ Materials Science Division, Argonne National Laboratory, Argonne, IL 60439, USA \\ ${ }^{4}$ CRISMAT-ISMRA, 14050 Caen Cedex, France
}

Abstract: We have magnetically imaged interlayer Josephson vortices emerging grafrat to 1 the planes of single crystals of the organic superconductor $\kappa$-(BEDT-TTF) $)_{2} \mathrm{Cu}(\mathrm{NCS})_{2}$. and the single layer cuprate high- $\mathrm{T}_{c}$ superconductors $\mathrm{Tl}_{2} \mathrm{Ba}_{2} \mathrm{CuO}_{6+8}(\mathrm{Tl}-2201)$ and ( $\mathrm{Hg}$, $\mathrm{Cu} \mathrm{B}$ de (Hg-1201), using a scanning Superconducting Quantum Interference Device (SQUI microscope. These images provide a direct measurement of the interlayer penetration depth, which is approximately $63 \mu \mathrm{m}$ for $\kappa$-(BEDT-TTF) $)_{2} \mathrm{Cu}(\mathrm{NCS})_{2}, 18 \mu \mathrm{m}$ for Tl-2201 and $8 \mu \mathrm{m}$ for $\mathrm{Hg}-1201$. The lengths for the cuprates are about a factor of 10 larger than originally predicted by the interlayer tunneling model for the mechanism of superconductivity in layered compounds, indicating that this mechanism alone cannot account for the high critical temperatures in these materials.

Key words: interlayer, vortex, SQUID, magnetic, microscopy

Many unconventional superconductors, notably cuprates and some organics, have a strongly anisotropic layered structure. The layered superconductors are commonly modeled using the Lawrence-Doniach model[1], as a stack of "conventional" superconducting layers with Josephson coupling between the layers. The strength of the interlayer coupling is an important parameter in any phenomenological description of these materials, but it can be difficult to measure. We discuss in this paper a direct way to measure this interlayer coupling, by imaging vortices trapped parallel to, and screened by, the superconducting layers. The magnetic "shape" of these vortices is directly related to the strength of the interlayer coupling, through the interlayer penetration depth, $\lambda_{1}=\left(c \Phi_{0} / 8 \pi^{2} \mathrm{~s} J_{0}\right)^{1 / 2}$ [2], where $J_{0}$ is the interlayer critical current density, $c$ is the speed of light, $\Phi_{0}=h c / 2 e$ is the superconducting flux quantum, $h$ is Planck's constant, $e$ is the charge on the electron, and $s$ is the interlayer spacing. Clem and Coffey derived expressions for the structure of vortices parallel to the layers (Fig. 1a), called "interlayer Josephson vortices", in the context of the Lawrence-Doniach model[2]. Except at the smallest length scales, these vortices are identical to vortices in an anisotropic London model. Kogan and Clem [3] calculated the magnetic fields from an anisotropic vortex extending above a superconducting surface. By comparing these expression with our measurements, we get quantitative values for the interlayer penetration depths. 


\section{DISCLAIMER}

This report was prepared as an account of work sponsored by an agency of the United States Government. Neither the United States Government nor any agency thereof, nor any of their employees, make any warranty, express or implied, or assumes any legal liability or responsibility for the accuracy, completeness, or usefuiness of any information, apparatus, product, or process disclosed, or represents that its use would not infringe privately owned rights. Reference herein to any specific commercial product, process, or service by trade name, trademark, manufacturer, or otherwise does not necessarily constitute or imply its endorsement, recommendation, or favoring by the United States Government or any agency thereof. The views and opinions of authors expressed herein do not necessarily state or reflect those of the United States Government or any agency thereof. 


\section{DISCLAIMER}

Portions of this document may be illegible in electronic image products. Images are produced from the best available original document. 


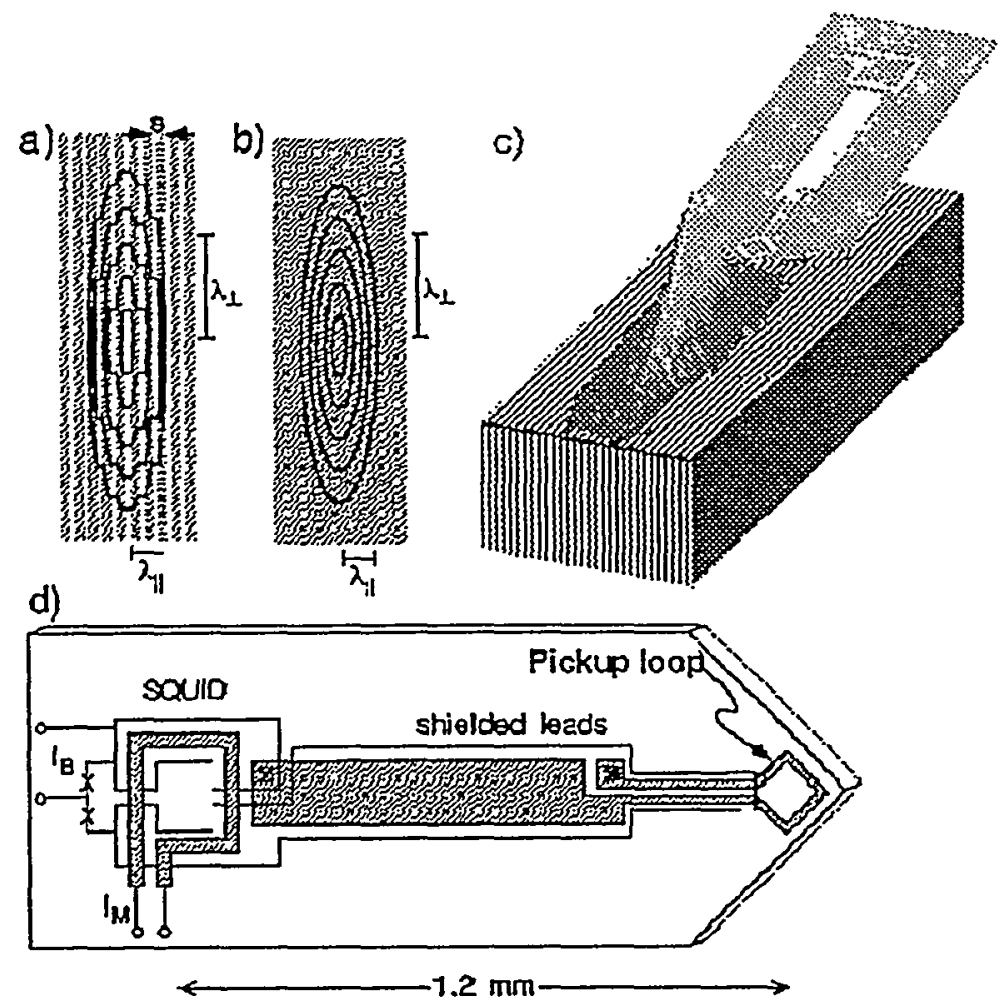

Fig 1. (a) Sketch of the current flow, equivalent to contours of constant magnetic field, for an isolated interlayer Josephson vortex with its axis parallel to the planes. The gray regions indicate superconducting layers with Josephson coupling between the layers. The interlayer spacing $s$, in-plane penetration depth $\lambda_{\|}$and interlayer penetration depth $\lambda_{\perp}$ are shown. b) For length scales large compared to $s$, the vortex structure becomes indistinguishable from a vortex in a continuous anisotropic London model. c) Sketch of the measurement geometry showing the pickup loop nearly parallel to the edge of a single crystal (not drawn to scale) with a 3-d rendering of scanning SQUID microscope data of an interlayer vortex in Tl-2201 superimposed. d) Schematic of the SQUID sensor. The signal is proportional to changes in the amount of magnetic flux through the pickup loop.

Our measurements were made with a scanning SQUID microscope [4], in which a sample is scanned relative to a superconducting pickup loop oriented approximately parallel to the sample surface (Fig. 1c). At any given position, the magnetic flux through the pickup loop, $\Phi_{s}$, is the integral of the $z$-component of the magnetic field over the area of the pickup loop. The data are represented as intensity maps of $\Phi_{s}$ vs. the position of the pickup loop in the $x, y$ plane. The pickup loop is fabricated with well-shielded leads to an integrated niobium SQUID (Fig. 1d).

The crystal growth of our $\kappa$-(BEDT-TTF) $)_{2} \mathrm{Cu}(\mathrm{NCS})_{2}[5], \mathrm{Tl}_{2} \mathrm{Ba}_{2} \mathrm{CuO}_{6+\delta}$ [6], and $(\mathrm{Hg}, \mathrm{Cu}) \mathrm{Ba}_{2} \mathrm{CuO}_{4+s}[7]$ single crystals has been described elsewhere. The organic superconducting crystals were mounted with vacuum grease with the highly conducting planes perpendicular to the 


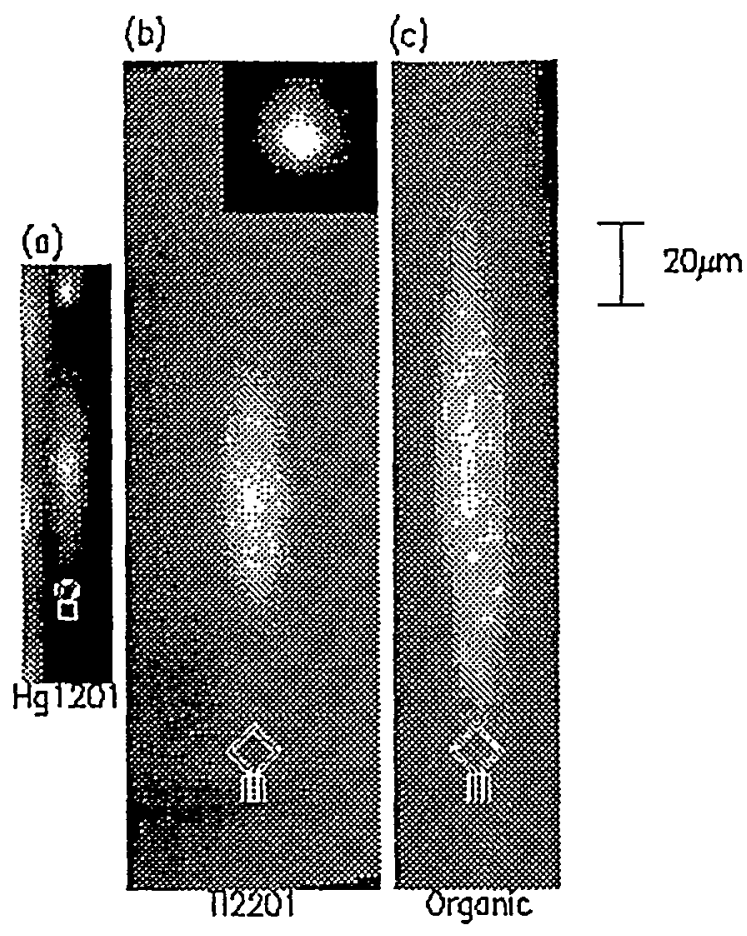

Fig.2 Scanning SQUID microscope images of interlayer Josephson vortices emerging parallel to the planes from single crystals of (a) $(\mathrm{Hg}, \mathrm{Cu}) \mathrm{Ba}_{2} \mathrm{CuO}_{4+\delta}, \quad$ (b) $\mathrm{Tl}_{2} \mathrm{Ba}_{2} \mathrm{CuO}_{6+\delta}$, and (c) $\kappa$-(BEDT-TTF $)_{2} \mathrm{Cu}(\mathrm{NCS})_{2}$. Scaled schematic drawings of the SQUID pickup loops used for each image are superimposed on the images: an octagonal loop $4 \mu \mathrm{m}$ in diameter was used for (a), and square pickup loops $8.2 \mu \mathrm{m}$ on a side were used for (b) and (c). Images of vortices emerging perpendicular to the planes of a single crystal of $\mathrm{YBa}_{2} \mathrm{CuO}_{7+\delta}$ using the same types of pickup loops are superimposed at the top of the images (a) and (b). The images are aligned so that the highly conducting planes are vertical. The vortex images are resolution limited horizontally (perpendicular to the planes), but have a well defined spatial extent vertically (parallel to the planes). The length of the vortices parallel to the planes is set by the interlayer penetration depth.

scan plane. The Hg-1201 and Tl-2201 crystals were embedded in epoxy in the same orientation and polished to expose a plane perpendicular to the $c$-axis direction.

Both sample and SQUID were immersed in liquid helium at $4.2 \mathrm{~K}$ in a magnetically shielded cryostat. A small magnet was used to adjust the component of the magnetic field perpendicular to the scan plane so that only a few well separated vortices were trapped in the crystal face. In the organic superconductors, there is an inhomogeneous magnetic background in addition to well defined vortices. This takes the form of "rippling" of the fields parallel to the layered planes. We speculate that these rippling features result from penetration of the external field on a length scale which is longer than the intrinsic interlayer penetration depth, and that these regions with weak coupling result from mechanical or chemical defects. In addition, the trapped positions of vortices tend to be inhomogeneous, as opposed to scattered uniformly throughout the crystal face, as is seen for vortices trapped with their axes perpendicular to the planes. This non-uniform trapping 
a)

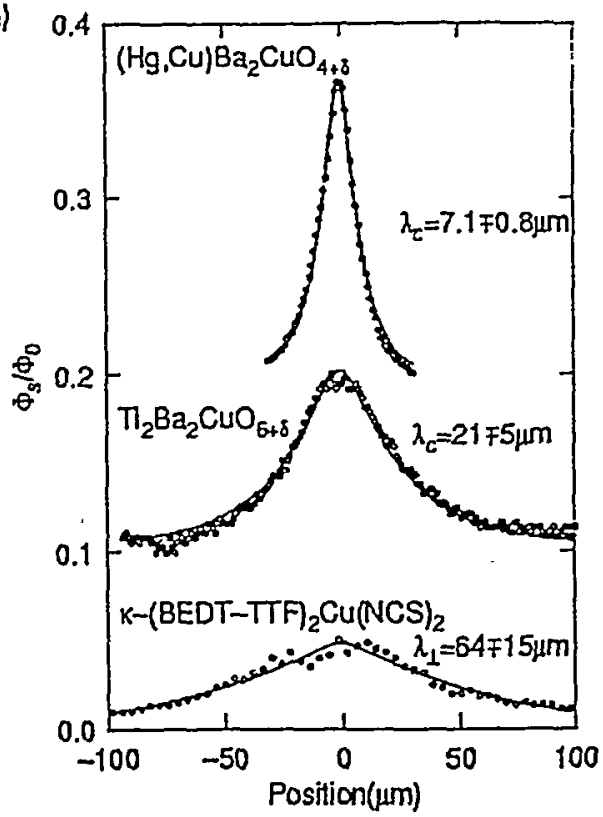

b)

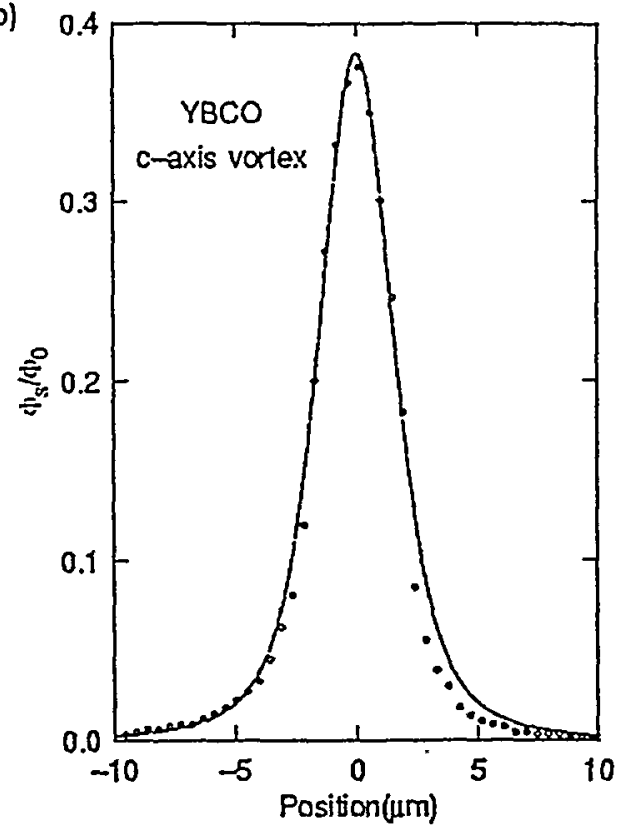

Fig. 3 a) Cross-sectional data through the images of Fig. 2, along vertical lines through the centers of the vortices and parallel to the highly conducting planes. The open circles are the data, and the lines are fits to this data using the theory of Ref. [3], with the height $z$ of the pickup loop above the sample surface, and the interlayer penetration depth $\lambda_{c}$ or $\lambda_{\perp}$ as the two fitting parameters. The organic superconductor and Tl-2201 data were taken with a square pickup loop $8.2 \mu \mathrm{m}$ on a side, and the $\mathrm{Hg}-1201$ data was taken with an octagonal loop $4 \mu \mathrm{m}$ on a side. b) For comparison, a $c$-axis Abrikosov vortex in YBCO, also taken with a $4 \mu \mathrm{m}$ octagonal loop. Note the change in the horizontal axis scale.

is a source for concern, as one could imagine that vortices trap in regions with exceptionally weak interplane coupling, so that our measurements might overestimate the intrinsic penetration depths. However, measurements of the Josephson plasma resonance, which average over large regions of the sample, are in good agreement with our measurements for Tl-2201[15].

A survey of our images of interlayer vortices in several superconductors is shown in Fig. 2. Cross-sections through these images, in the vertical direction parallel to the conducting planes, are shown as the open circles in Fig. 3. The lines in Fig. 3 are fits to these cross-sections as follows: The $z$ component of the magnetic field of an interlayer vortex (modelled as an anisotropic Ginzburg-Landau vortex) above the superconducting surface (including spreading of the vortex as it approaches the surface), is given by:

$h(\vec{r}, z)=-\int \frac{d^{2} \vec{k}}{(2 \pi)^{2}} k \phi(\vec{k}) e^{i \vec{k} \cdot p-k z}$,

where 
$\phi(\vec{k})=-\frac{\phi_{0}\left(1+m_{1} k_{x}^{2}\right)}{m_{3} a_{3}\left[m_{1} k_{x}^{2} a_{3}\left(k+a_{1}\right)+k a_{3}+k_{y}^{2}\right]}$,

$\alpha_{1}=\left(\left(1+m_{1} k^{2}\right) / m_{1}\right)^{1 / 2}, a_{3}=\left(\left(1+m_{1} k_{x}^{2}+m_{3} k_{y}^{2}\right) / m_{3}\right)^{1 / 2}, k=\left(k_{x}^{2}+k_{y}^{2}\right)^{1 / 2}, m_{1}=\lambda_{\|}^{2} / \lambda^{2}$, $m_{3}=\lambda_{\perp}^{2} / \lambda^{2}, \lambda=\left(\lambda_{\|}^{2} \lambda_{\perp}\right)^{1 / 3}$, where $\lambda_{\|}$is the (short) in-plane penetration depth, and $\lambda_{\perp}$ is the (long) interplane penetration depth. The fields are summed over the geometry of the pickup loop. The solid circles in Fig. 3 are the experimental data, the lines are the fits to the equations above. With the London penetration depth $\lambda_{\|}$fixed at $0.17 \mu \mathrm{m}$ for $\mathrm{Hg}-1201$ and $\mathrm{Tl}-2201$, and $0.52 \mu \mathrm{m}$ for the organic superconductor, the two fitting parameters are the height $z$ of the pickup loop above the surface, and $\lambda_{\perp}$. Fits of a number of such vortices for each crystal resulted in values of $\cdots \lambda_{c}=8 \pm 1 \mu \mathrm{m}$ for $(\mathrm{Hg}, \mathrm{Cu}) \mathrm{Ba}_{2} \mathrm{CuO}_{4+\delta}[9], \lambda_{c}=18 \pm 3 \mu \mathrm{m}$ for $\mathrm{Tl}_{2} \mathrm{Ba}_{2} \mathrm{CuO}_{6+\delta}[6,3]$, and $\lambda_{\perp}=63 \pm$ $15 \mu \mathrm{m}$ for $\kappa-(\mathrm{BEDT}-\mathrm{TTF})_{2} \mathrm{Cu}(\mathrm{NCS})[8]$.

These values of $\lambda_{\perp}$ are of special interest as a test for a candidate mechanism for superconductivity in these materials. It has been proposed that non-Fermi liquid behavior in the normal state may drive the superconducting transition because of a change in the interlayer coupling between the two states[10,11,12,13]. This class of mechanisms is known as the Interlayer Tunneling (ILT) model. In the simplest version of the ILT model, the superconducting condensation energy, $E_{c}$, is supplied entirely by a change in the kinetic energy perpendicular to the planes. The model therefore requires a relationship between the interlayer penetration depth and the condensation energy $\lambda_{I L T}=\left(\frac{m c^{2}}{E_{c}} \frac{a_{o} A}{4 \pi s}\right)^{1 / 2}$, where $E_{c}=H_{c}^{2} A s / 8 \pi$ is the condensation energy per formula unit, $H_{c}$ is the thermodynamic critical field, $A$ is the area per formula unit, $m$ is the mass of the electron, and $a_{o}$ is the Bohr magneton[12-14]. The original estimates of the condensation energy from specific heat measurements lead to $\lambda_{I L T} \simeq 1 \mu \mathrm{m}$ for $\mathrm{Tl}-2201$ and $\mathrm{Hg}-1201$, and $\lambda_{I L T} \simeq 10 \mu \mathrm{m}$ for $\kappa$-(BEDT-TTF) $)_{2} \mathrm{Cu}(\mathrm{NCS})$. This would imply that the amount of energy available to the superconducting transition from the interlayer coupling is about 2 orders of magnitude smaller than the condensation energy. Recently Chakravarty and co-workers have suggested that the condensation energies can be much lower than originally estimated due to fluctuation effects[16]. When these effects are taken into account, their values for $\lambda_{I L T}$ are within a factor of two of our measurements for $\lambda_{c}$ for T1-2201. However, this still implies that the ILT mechanism alone cannot account for the large critical temperatures in these materials.

An alternate view of interlayer Josephson coupling results if we start with the noimal-state conductivity, and assume either specular or diffuse interlayer pair transport. For diffuse pair transport (parallel momentum not conserved $[17,18,19]$, the Josephson current density between two identical superconducting sheets at $T=0$ is given by $J_{o}=\pi \Delta_{0} / 2 e R_{\perp}[20]$, where $\Delta_{0}$ is the zero temperature energy gap, and $R_{\perp}$ is the normal state resistance per plane perpendicular to the planes. If we take measured values for $R_{\perp}$ just above $T_{c}$, and the BCS value $\Delta_{0}=1.76 k_{B} T_{c}$, we find values of $\lambda_{\perp} \sim 20 \mu m$ for $\kappa$-(BEDT-TTF) $)_{2} \mathrm{Cu}(\mathrm{NCS}), \lambda_{\perp} \sim 6 \mu \mathrm{m}$ for Tl-2201, and $\lambda_{\perp} \sim 8 \mu \mathrm{m}$ for $\mathrm{Hg}-1201$. The application of this model requires at least two assumptions: that $R_{\perp}$ is temperature independent below $T_{c}$, and that the gap is s-wave. One would expect the coupling for a d-wave gap (as is believed to be true for the cuprates) to be smaller than estimated here: in fact, the interplane coupling is expected to be zero for a isotropic d-wave superconductor with

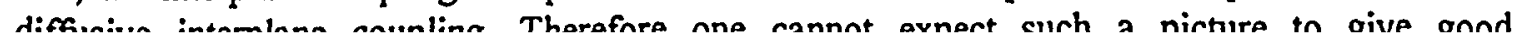


quantitative agreement with experiment, but it does agree to within a factor of roughly 3 for all of our measurements, and in fact works well for a number of the cuprate superconductors[21].

In conclusion, magnetic imaging of interlayer vortices with the scanning SQUID microscope is a powerful tool for directly measuring the interlayer penetration depth. Measurements on an organic superconductor and two single-plane cuprate superconductors indicate that the interlayer tunneling mechanism alone cannot account for the high critical temperatures observed in these materials.

We would like to thank M.B. Ketchen for the design, and M. Bhushan for the fabrication, of the SQUIDs used in our microscope. We would also like to acknowledge useful conversations with -P.W. Anderson, S. Chakravarty, A.J. Leggett, D.J. Scalapino, C. Panagopoulos, D. van der Marel, V.G. Kogan, and J. Clem. K.A. Moler would like to acknowledge the support of an R.H. Dicke post-doctoral fellowship, and NSF funding through the MRSEC award to Princeton University (DMR 97-00362).

[1] Lawrence WE, and Doniach S (1971) In E. Kanda (ed) Proceedings of the 12th International Conference on Low Temperature Physics. Academic Press. Kyoto. pp 361-62

[2] Clem JR and Coffey MW (1990) Phys. Rev. B 42:6209-16; Clem JR, Coffey MW, and Haro Z (1991) Phys. Rev. B 44:2732-38; Gurevich A, Benkraouda M, and Clem JR (1996) Phys. Rev. B 54:3196-206

[3] Kirtley JR, Kogan V, Clem JR, and Moler KA, Phys. Rev. B in press

[4] Kirtley JR et al. (1995) Appl. Phys. Lett. 66:1138-40

[5] Carlson KD et al. (1988) Inorg. Chem. 27:965-67

[6] Moler KA, Kirtley JR, Hinks DG, Li TW, and Xu M (1998) Science 279:1193-96

[7] D. Pelloquin et al. (1997) Physica C 273:205-212

[8] Kirtley JR, Moler KA, Schlueter JA, and Williams JM, submitted to J. Phys. Condens. Matt.

[9] Kirtley JR, Moler KA, Villard G, and Maignan A (1998) Phys. Rev. Lett. 81:2140-2143

[10] Wheatley J, Hsu T, Anderson PW (1988) Nature 333:121-21

[11] Anderson PW (1991) Physica C 185:11-16; (1991) Phys. Rev. Lett. 67:660-63; (1992) Science 256:1526-31

[12] Chakravarty S, Sudbo A, Anderson PW, and Strong S (1993) Science 261:337-40

[13] Anderson PW (1995) Science 268:1154-55

[14] Leggett AJ (1996) Science 274:587-89

[15] Tsvetkov AA (1998) Nature 395:360-62

[16] Chakravarty S, Kee H.Y. and Abrahams E., preprint

[17] Graf MJ, Rainer D, Sauls JA (1993) Phys. Rev. B 47:2089-98

[18] Rojo AG and Levin K (1993) Phys. Rev. B 48:6861-64

[19] Hirschfeld PJ, Quinlan SM, Scalapino DJ (1997) Phys. Rev. B 55:2742-47

[20] Ambegaokar V and Baratoff A (1963) Phys. Rev. Lett. 10:456-59; 11:401(E)

[21] Basov DN et al. (1994) Phys. Rev. B 50:3511-14 